\title{
Actualización de las Recomendaciones sobre la logística de uso de la ecocardiografía durante la pandemia de COVID-19. Recomendaciones de la Sociedad Española de Imagen Cardíaca
}

Miguel Ángel García Fernández* Pedro María Azcárate Agüero**

María Carolina Cabrera Schulmeyer***
José Luis del Pozo León $* * * *$

José Juan Gómez de Diego*****

\section{Correspondencia}

Miguel Ángel García Fernández garciafernandez@ecocardio.com

\footnotetext{
* Catedrático de Imagen Cardíaca. Universidad Complutense de Madrid. Madrid. España

** Departamento de Cardiología. Hospital San Pedro de Logroño. La Rioja. España

*** Departamento de Anestesia de la Universidad de Valparaíso. Chile

**** Director del Servicio de Microbiología Clínica y del Área de Enfermedades Infecciosas de la Clínica Universidad de Navarra. España

***** Departamento de Imagen Cardíaca del Servicio de Cardiología. Hospital Universitario Clínico San Carlos. Madrid. España
}

Recibido: 30/05/2020

Aceptado: 30/05/2020

En línea: 31/07/2020

Citar como: García-Fernández MA, Azcaráte-Agüero PM, Cabrera-Schulmeyer MC, Del Pozo-León JL, Gómez-de Diego JJ. Actualización de las Recomendaciones sobre la logística de uso de la ecocardiografía durante la pandemia de COVID-19. Recomendaciones de la Sociedad Española de Imagen Cardíaca. Rev Ecocar Pract (RETIC). 2020 (Jul); 3 (2): 1-5. doi: 10.37615/retic.v3n2a2.

Cite this as: García-Fernández MA, Azcaráte-Agüero PM, Cabrera-Schulmeyer MC, Del Pozo-León JL, Gómez-de Diego JJ. Update of the Recommendations of the Logistic Use of Echocardiography during the COVID-19 Pandemic. Rev Ecocar Pract (RETIC). 2020 (Jul); 3 (2): 1-5. doi: 10.37615/retic.v3n2a2.

\section{Introducción}

El virus COVID-19 (SARS-Cov-2) es una cepa de la familia de coronavirus que no se había identificado previamente en humanos. Los coronavirus provocan distintos tipos de procesos patológicos que van desde el resfriado común hasta enfermedades más graves, como insuficiencia respiratoria aguda grave. El miércoles 11 de marzo de 2020, el Director General de la Organización Mundial de la Salud, el Dr. Tedros Ghebreyesus, declaró la situación de COVID-19 como una pandemia global.

El virus se transmite fácilmente de persona a persona cuando se tiene contacto cercano con un enfermo o a través de fómites.

Los datos de afectación cardíaca en pacientes ingresados son muy recientes En una serie de 138 pacientes hospitalizados con COVID-19, el 16,7\% desarrollaron arritmias y el 7,2\% experimentó una lesión cardíaca aguda, además de otras complicaciones relacionadas con COVID-19(1). Hay publicados casos aislados de insuficiencia cardíaca de inicio agudo, infarto de miocardio, miocarditis y paro cardíaco. Al igual que con cualquier enfermedad aguda, una mayor demanda cardiometabólica puede precipitar complicaciones cardíacas.

Resulta evidente que, dadas las características de nuestra especialidad, en algunos entornos específicos, el equipo de atención cardiovascular (incluidos médicos, enfermeros, técnicos, etc.) puede tener una capacitación y experien- cia limitadas en el manejo agudo de esta enfermedad pandémica. En una reciente comunicación del American College of Cardiology (ACC)(2) se insiste en que "la transmisión rutinaria de COVID-19 a los trabajadores de la salud sugiere que las precauciones diarias de prevención de las enfermedades infecciosas son insuficientes y que los trabajadores de la salud en las zonas afectadas deben estar preparados para adoptar medidas de protección personal adicional". Como ya se ha dicho, el virus SARS-Cov-2 se contagia fácilmente sobre todo si se está muy cerca de los pacientes, como ocurre durante la realización de un estudio ecocardiográfico. Por tanto, los profesionales que realizan estas exploraciones están especialmente en riesgo de infectarse por un virus con un alto grado de contagio y una alta mortalidad y morbilidad.

\section{Escenarios clínicos}

Lo primero que se debe tener en cuenta es que todo paciente al que se le realice un estudio ecocardiográfico debe portar una máscara de filtración simple o FFP2.

Los escenarios clínicos a los que se va a hacer frente son los siguientes. Por un lado, hay que tener que seguir realizando estudios en pacientes con COVID-19 en los que la ecocardiografía puede ser básica cuando exista sospecha de afectación cardíaca, fallo cardíaco, arritmia, cambios electrocardiográficos o cardiomegalia. La realización del estudio es compleja desde el punto de vista de la protección, dado que, como ya se ha dicho, resulta imposible mantener una distancia mínima de seguridad con el paciente, y el examen 
ecocardiográfico se convierte en uno de los de mayor riesgo para los profesionales de la salud. En los pacientes con COVID-19 que necesiten un estudio en el laboratorio de ecocardiografía, debería habilitarse una sala y un circuito específico: (circuito COVID). De no ser así, sería recomendable llevar el ecocardiógrafo a donde se encuentren los pacientes, bien utilizando equipos portátiles o, si es posible, utilizando equipos que puedan estar disponibles en distintas áreas del hospital (urgencias, intensivos, quirófano...), con el objetivo de minimizar el riesgo de contagio entre pacientes. Cuando se acercan los equipos de ecocardiografía a los pacientes enfermos es importante que el médico que realiza la exploración tenga la formación necesaria en ecocardiografía y que los equipos tengan la capacidad de almacenar las imágenes obtenidas para que puedan ser reevaluadas en el laboratorio de ecocardiografía si fuese necesario.

Por otro lado, en la fase de vuelta a la normalidad de los distintos laboratorios de ecocardiografía, se va a hacer frente a pacientes en los que no se sabe si la infección está presente. Probablemente, éstos sean los estudios donde haya que pensar mejor cómo actuar y protegerse. No sólo por la posibilidad de que el virus se transmita al personal sanitario, sino porque los laboratorios pueden convertirse en un foco de transmisión del virus entre pacientes y al personal de salud. Los pacientes que se estudian de forma regular son más vulnerables al virus ya que presentan, en muchas ocasiones, comorbilidades importantes: pacientes oncológicos, ancianos, inmunodeprimidos, con afectación cardíaca, etcétera ${ }^{(3)}$

\section{Protección}

La Sociedad Española de Imagen Cardíaca (SEIC), al igual que otras sociedades científicas, emitió unas recomendaciones con fecha 17 de marzo de 2020(4). Dada la rápida evolución de la pandemia y la situación de nuevos escenarios como el fin de la fase intensa de confinamiento, la SEIC ha decidido actualizar el documento consensuado insistiendo en la importancia de realizar un estudio con las máximas garantías.

Por un lado, sigue siendo muy importante establecer la adecuada indicación del mismo y por otro, como ya se ha comentado, es vital realizar los estudios en un ambiente de trabajo seguro tanto para proteger al personal sanitario como para evitar la transmisión del virus entre pacientes. Y es que, a pesar de que se sigue insistiendo en la protección del personal sanitario, la realidad es que éste sigue estando muy expuesto. En concreto, con fecha 21 de marzo de 2020, la revista Lancet publicaba que el $20 \%$ del personal sanitario italiano se había infectado ${ }^{(5)}$.

Por otro lado, hay que tener en cuenta que cuando el profesional sanitario no se siente protegido, se encuentra aún más expuesto al estrés físico y mental. Esto puede provocar que los errores diagnósticos a la hora de interpretar los estudios se multipliquen ${ }^{(6)}$.

En resumen, una inadecuada protección puede llevar a un mayor contagio entre los profesionales sanitarios, a que la enfermedad se siga propagando desde los laboratorios y a errores diagnósticos que pueden resultar fatales en el manejo de los pacientes. Por tanto, deben implementarse las medidas de protección que se detallan en este documento, lavarse adecuadamente las manos tras la realización de cada estudio y desechar adecuadamente el material de protección indicado.

\section{Indicaciones}

En los dos escenarios clínicos que se pueden dar, paciente con COVID-19 o paciente asintomático del que no se conoce si está infectado o no, la SEIC insiste en la posibilidad de que el cardiólogo especialista en imagen cardíaca pueda rechazar la realización de estudios con una indicación inapropiada, limitando en lo posible el acceso a áreas infectadas. Igualmente recomienda el uso de equipos fijos que se movilicen lo menos posible entre las diferentes áreas hospitalarias. Además, es muy importante tratar de disminuir el tiempo que se dedica a la realización de cada estudio, por eso se sigue proponiendo que se realicen "estudios orientados o focalizados". También es importante que estos estudios se realicen por profesionales con experiencia, con el objetivo, por un lado, de limitar el tiempo de exposición y, por otro, para que se obtenga toda la información necesaria y evitar así tener que volver a repetir el mismo ${ }^{(7)}$. No sólo es importante que el estudio esté bien indicado ${ }^{(8,9)}$. Además, su realización debe limitarse sólo a actuaciones en las que el paciente vaya a beneficiarse clínicamente de este acto clínico y que éste produzca cambios en las conductas terapéuticas. Por ejemplo, debe evitarse la realización de ecocardiografías seriadas en un mismo paciente si no ha habido cambios clínicos.

Por tanto, la realización de cada estudio debe ponderarse adecuadamente; se debe revisar su indicación y si su realización va a ser beneficiosa para el paciente.

También se debe revisar si el paciente tiene estudios previos:

- Para evitar la necesidad de volver a realizar la prueba si el estudio es reciente y no ha habido cambios clínicos.

- Para conocer detalladamente cómo estaba el corazón del paciente antes de realizar un nuevo estudio.

- Para saber si se va a necesitar la utilización de contraste en casos de malas ventanas previas u otro tipo de medicación.

Reducir los tiempos de realización de las pruebas es importante para evitar la transmisión de la enfermedad.

En casos seleccionados, el estudio ecocardiográfico deberá ser detallado y completo.

\section{Procedimientos de mayor riesgo}

Además de la realización de estudios en pacientes con enfermedad conocida, de los procedimientos que se realizan en los laboratorios de ecocardiografía, dos son de especial riesgo:

- Ecocardiografía transesofágica (ETE). Es de especial riesgo porque puede provocar aerosoles. Por ello, se recomiendan unas medidas de protección individual más estrictas y se considera que se debe realizar una determinación de PCR para saber si el paciente está infectado antes de realizar la prueba. Además, hay que ser más estrictos al establecer su indicación y realizar un procedimiento diagnóstico alternativo de menor riesgo si está disponible; por ejemplo, se recomienda realizar una TC cardíaca para detectar trombos intracavitarios en vez de una ETE, ya que está demostrado que tiene una especificidad y sensibilidad superponibles ${ }^{(10)}$.

- Ecocardiografía de estrés físico. Es el otro procedimiento de especial riesgo y se remite al lector a su apartado específico más adelante.

\section{Protocolos de protección}

\section{Estudios realizados en pacientes ambulatorios (Figuras 1 y 2)}

En estos pacientes debe hacerse una búsqueda activa de la enfermedad mediante PCR, si van a ser sometidos a un procedimiento que genere aerosoles como son las ETE y las ecocardiografías de estrés físico. 
En función de la capacidad de cada laboratorio, se puede dedicar una máquina y una sala para pacientes enfermos (circuito COVID).

\section{Ecocardiografías transesofágicas (Figura 3)}

Como ya se ha mencionado con anterioridad, este procedimiento es susceptible de generar aerosoles. Por ello, su indicación debe estar bien establecida y también puede ser valorada junto con el médico que la solicita y eventualmente diferirla, si su indicación no es perentoria en función de la fase de la pandemia en la que cada país se encuentre.

En los casos en los que se pueda obtener la información médica mediante otra prueba, se recomienda no realizar esta prueba y utilizar, por ejemplo, TC cardíaca para la búsqueda activa de trombos intracavitarios antes de realizar una cardioversión.
Ecocardiografías de estrés (Figura 3)

La ecocardiografía de estrés físico puede suponer un procedimiento de mayor riesgo. Por tanto, esta prueba puede diferirse en función de la fase de la pandemia en la que cada país se encuentre. El paciente va a tener que realizar respiraciones rápidas y profundas pudiendo generar aerosoles en un espacio cerrado. Dado que hoy en día la TC cardíaca es una técnica muy precisa en el diagnóstico de pacientes con dolor torácico y probabilidad intermedia de tener enfermedad coronaria, desde la SEIC se recomienda utilizar esta prueba ${ }^{(11)}$. En función de cada paciente o de las posibilidades del centro también puede realizarse una ecocardiografía de estrés farmacológico. En caso de necesitar la realización de una ecocardiografía de estrés físico para el estudio de enfermedad cardíaca no coronaria ${ }^{(12)}$ es recomendable realizar una PCR al paciente para conocer si está infectado y tomar unas medidas de protección adecuadas.

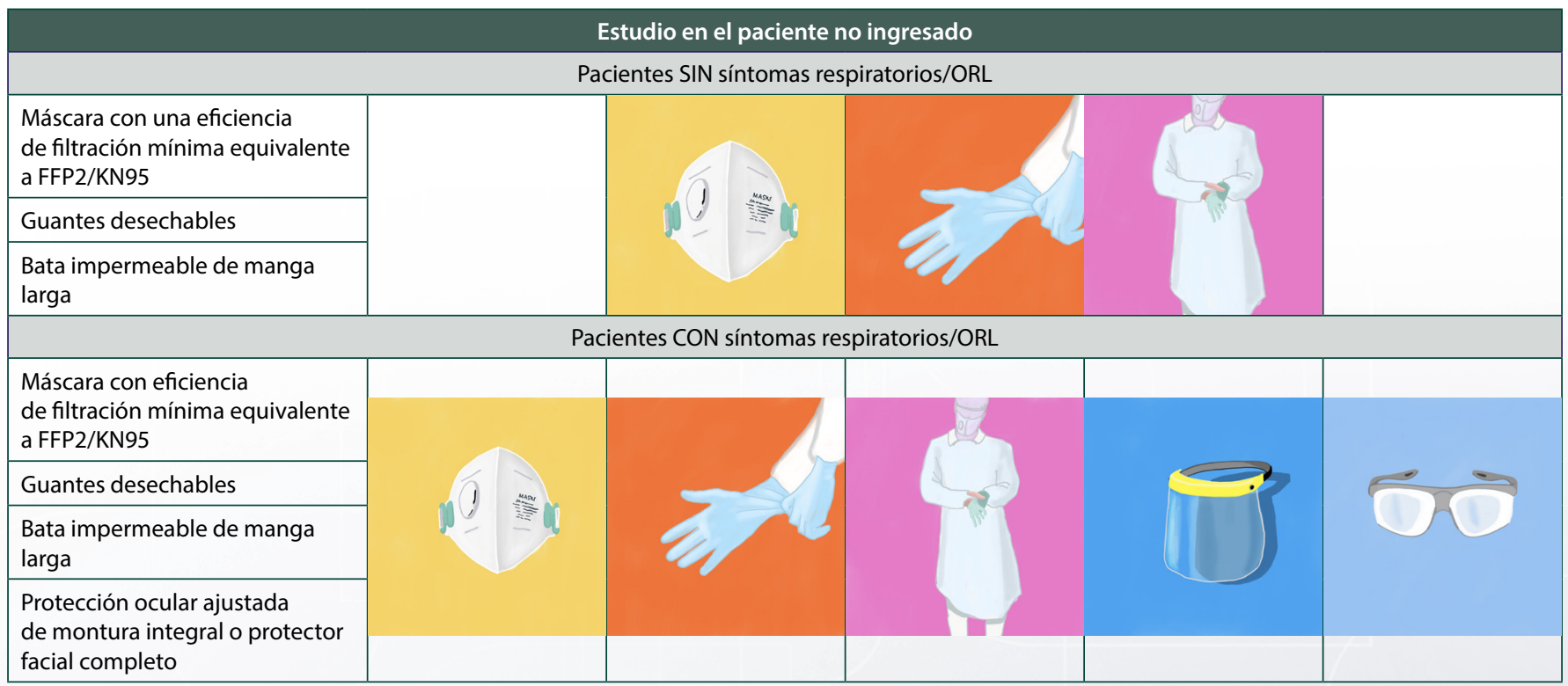

Figura 1. Esquema de las precauciones que deben tomarse para realizar una ecocardiografía a un paciente con sospecha de infección por COVID-19

Estudio en el paciente ingresado

Pacientes SIN síntomas respiratorios/ORL

Máscara con una eficiencia de filtración mínima equivalente a FFP2/KN95

Guantes desechables

Bata impermeable de manga larga

$\begin{aligned} & \text { Máscara con eficiencia } \\
& \text { de filtración mínima equivalente } \\
& \text { a FFP2/KN95 }\end{aligned}$
\begin{tabular}{ll|l|l|l|}
\hline Guantes desechables \\
Bata impermeable de manga \\
larga
\end{tabular}
$\begin{aligned} & \text { Protección ocular ajustada } \\
& \text { de montura integral o protector } \\
& \text { facial completo }\end{aligned}$

Figura 2. Esquema de las precauciones que deben tomarse para realizar una ecocardiografía a un paciente con sospecha de infección por COVID-19 


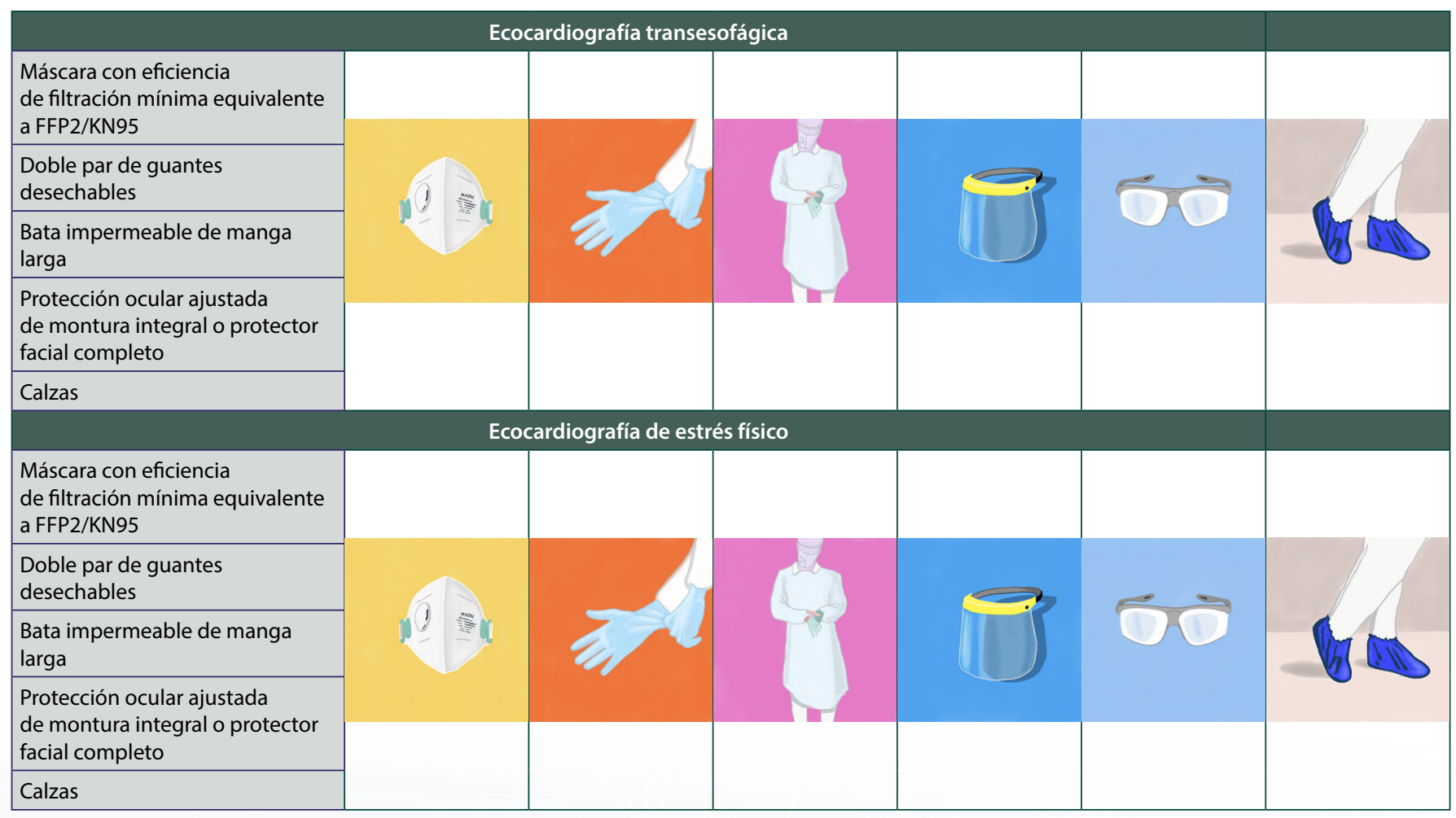

Figura 3. Esquema de las precauciones que deben tomarse para realizar una ecocardiografía de alto riesgo a un paciente con sospecha de infección por COVID-19

\section{Protección y limpieza del ecocardiógrafo y de la sonda transesofágica ${ }^{(13)}$}

Los coronavirus son virus envueltos por una capa lipídica que los hace ser especialmente sensibles a los desinfectantes de uso habitual en el medio sanitario. La evidencia disponible ha demostrado que los virus se inactivan de manera efectiva con procedimientos de desinfección adecuados que incluyen el uso de desinfectantes comunes para uso hospitalario. El uso de estos desinfectantes debe tener un tiempo de contacto adecuado. De acuerdo con lo sugerido por la OMS, "la limpieza a fondo de las superficies ambientales con agua y detergente seguida de la aplicación de desinfectantes comúnmente utilizados a nivel hospitalario" son procedimientos efectivos y suficientes.

Son múltiples las medidas que se pueden tomar para proteger los equipos; utilización de fundas para las sondas, empleo de medidas de barrera entre el paciente y el equipo, evitar el uso de electrodos, etc., para evitar así la transmisión de la enfermedad. Es recomendable utilizar también gel individual en los pacientes COVID +, ya que el envase de gel puede contaminarse.

La limpieza ordinaria del ecocardiógrafo se puede realizar con gasas empapadas en soluciones de alcohol al 70\%, comúnmente utilizadas en un entorno hospitalario.

En el caso de las sondas transtorácicas expuestas a patógenos, la desinfección debe ser efectiva contra todos los agentes transmisibles y se puede utilizar una gran cantidad de productos:

- Con base de hipoclorito sódico. Por ejemplo, solución de lejía al 10\% para uso doméstico con hipoclorito sódico activo al 0,6\%, aproximadamente.
- Con base de amoníaco cuaternario (QUAT). Por ejemplo, productos que contienen solución de cloruro de amonio de xbencilo n-alquilo, donde $x$ puede ser cualquier grupo funcional orgánico como etil- y metil-, etc:; la concentración total para el uso debe ser inferior al 0,8\% para todos los QUAT enumerados.

- Con base de peróxido de hidrógeno acelerado (peróxido de hidrógeno al 0,5\% máximo).

- Con base de alcohol o alcohol y amoníaco cuaternario (QUAT). El contenido del producto de alcohol no puede exceder de $70 \%$.

Sin embargo, dado que no todas las soluciones de limpieza son compatibles con los transductores disponibles, se recomienda consultar los manuales de mantenimiento y limpieza de cada equipo.

Para evitar contaminaciones en los geles ecográficos se aconseja cerrar su envase tras cada uso y no dejarlo abierto continuamente. El uso de dosis individuales de gel no está extendido en España pero, si están disponibles, se recomienda su uso. En caso de no disponer de las mismas se pueden utilizar jeringuillas de 10, 20 o $50 \mathrm{ml}$. cargadas de gel ecocardiográfico, a la hora de aplicarlo sobre el tórax del paciente y así evitar la transmisión del virus a través del gel.

Por último, hay que considerar que se deben proteger los equipos y las sondas mediante material desechable. Por ejemplo, fundas en el caso de las sondas transtorácicas o transesofágicas.

\section{Agradecimiento}

A Manuel Álvarez García por los dibujos utilizados en este documento 


\section{Bibliografía}

1. Wang D, Hu B, Hu C, et al. Clinical characteristics of 138 hospitalized patients with 2019 novel coronavirus-infected pneumonia in Wuhan, China. JAMA 2020; 323 (11): 1061-1069.

2. COVID-19 Clinical Guidance For the Cardiovascular Care Team document. Disponible en: ACC: https://www.acc.org/ /media/Non-Clinical/Files-PDFsExcel-MS-Word-etc/2020/02/S20028-ACC-Clinical-Bulletin-Coronavirus. pdf

3. Documento ad uso degli operatori di ecografia cardiovascolare per covid-19 SIEC. Disponible en: https://www.siec.it/documento-ad-uso-degli-operatori-di-ecografia-cardiovascolare-per-covid-19/

4. García Fernández MA, Cabrera Schulmeyer MA, Azcárate Agüero PM. Documento sobre el uso de la ecocardiografía en pacientes con COVID-19. Disponible en: https://ecocardio.com/documentos/covid-19/2064-documentouso-ecocardiografia-pacientes-covid19.html

5. COVID-19: protecting health-care workers. Lancet 2020; 21: 395.

6. Fraser AG. A manifesto for cardiovascular imaging: addressing the human factor. Eur Heart J Cardiovasc Imaging 2017; 18: 1311-1321.

7. ASE Statement on protection of patients and echocardiography service providers during the 2019 novel coronavirus outbreak. Endorsed by the American College of Cardiology. Disponible en: https://www.asecho.org/wp-content/uploads/2020/03/COVIDStatementFINAL4-1-2020_v2_website.pdf

8. Writing Group Members, Doherty JU, Kort S, Mehran R, et al. Appropriate use Criteria Task Force Multimodality Imaging in the assessment of cardiac structure and function in nonvalvular heart disease: A report of the American College of Cardiology Appropriate Use Criteria Task Force, American Association for Thoracic Surgery, American Heart Association, American So- ciety of Echocardiography, American Society of Nuclear Cardiology, Heart Rhythm Society, Society for Cardiovascular Angiography and Interventions, Society of Cardiovascular Computed Tomography, Society for Cardiovascular Magnetic Resonance, and the Society of Thoracic Surgeons. J Am Soc Echocardiogr 2019; 32: 553-579.

9. Doherty JU, Kort S, Mehran R, et al. ACC/AATS/AHA/ASE/ASNC/HRS/SCAI/ SCCT/SCMR/STS 2017 Appropriate Use Criteria for Multimodality Imaging in Valvular Heart Disease: A Report of the American College of Cardiology Appropriate Use Criteria Task Force, American Association for Thoracic Surgery, American Heart Association, American Society of Echocardiography, American Society of Nuclear Cardiology, Heart Rhythm Society, Society for Cardiovascular Angiography and Interventions, Society of Cardiovascular Computed Tomography, Society for Cardiovascular Magnetic Resonance, and Society of Thoracic Surgeons. J Am Soc Echocardiogr 2018; 31: 381-404

10. Guglielmo M, Baggiano A, Muscogiuri G, et al. Multimodality imaging of left atrium in patients with atrial fibrillation. J Cardiovasc Comput Tomogr 2019; 13: 340-346.

11. Knuuti J, Wijns W, Saraste A, et al. 2019 ESC Guidelines for the Diagnosis and Management of Chronic Coronary Syndromes. Eur Heart J 2020; 41: 407477.

12. Lancellotti P, Pellikka PA, Budts W, et al. The clinical use of stress echocardiography in non-ischaemic heart disease: Recommendations from the European Association of Cardiovascular Imaging and the American Society of Echocardiography. Eur Heart J Cardiovasc Imaging 2016; 17: 1191-1229.

13. Kampf G, Todt D, Pfaender S, Steinmann E. Persistence of coronaviruses on inanimate surfaces and their inactivation with biocidal agents. J Hosp Infect 2020; 104: 246-251. 